\title{
The Booster Application Facility (BAF) Beam transport Line of BNL-AGS Booster*
}

\author{
N. Tsoupas*, K.A. Brown, J. R. Cullen, Y.Y. I ee, A. J. Mc Nerney, P.H. Pile, T. Roser, \\ A. Soukas, and J.E. Tuozzolo \\ Brookhaven National Laboratory, Upton, NY 11973 USA
}

\section{Abstract}

An experimental facility ${ }^{1}$, to irradiate materials with energetic ion beams, has been proposed to be built at the Brookhaven National Laboratory. The BAF facility will mainly consist of the AGS-Booster slow extraction, of a beam transport line, and a target room. The beam transport line will transport the slow extracted beam of the AGS-Booster to the target location for the irradiation of various materials and specimens. A variety of ion beams like $\left(\mathrm{p},{ }^{28} \mathrm{Si},{ }^{56} \mathrm{Fe},{ }^{63} \mathrm{Cu},{ }^{197} \mathrm{Au}\right)$ in the energy range of 0.04 to $3.07 \mathrm{GeV} /$ nucleon will be transported by the $B A F$ line which is designed to provide variable beam spot sizes on the BAF target with sizes varying from 2.0 $\mathrm{cm}$ to $20.0 \mathrm{~cm}$ in diameter. The beam spot sizes will include $95 \%$ of the beam intensity with the beam distributed normally (Gaussian) on the target area. It is also possible by introducing magnetic octupoles at specified locations along the beam transport line, to modify the distribution on the BAF target and provide well confined beams with rectangular cross section and with uniform distribution on the target[1].

\section{INTRODUCTION}

The function of the AGS Booster synchrotron is to accelerate a variety of ion species (protons to $\mathrm{Au}$ ) to a maximum rigidity of $B \rho=7.5 \mathrm{~T} . \mathrm{m}$ for protons to $17.5 \mathrm{~T} . \mathrm{m}$ for $\mathrm{Au}$ ions and then inject the accelerated bunches into the AGS synchrotron which further accelerates the beam bunches to a rigidity of $\mathrm{BP}=100 \mathrm{Tm}$ (proton momentum equivalent $\mathrm{p}=29.9 \mathrm{GeV} / \mathrm{c}$ ). Subsequently the accelerated beam will be injected into the Relativistic Heavy Ion Collider (RHIC) which will accelerate and store the bunches to the desired energy for the beam-beam experiments. Since the storage cycle in the RHIC synchrotron may last many hours, all the pre-accelerators will be available during this time to provide high energy beams to other facilities at BNL. The proposed BAF facility shown in Figure 1 is one which will operate during the storage cycle of RHIC synchrotron. The construction of the BAF facility is scheduled for completion commissioning and operation by the year 2001.

\footnotetext{
* Work supported by the U.S. Department of Energy * email tsoupas@bnl.gov

${ }^{1}$ In this document, this facility will be referred to as the Booster Applications Facility (BAF).
}

\section{THE (BAF) BEAM TRANPORT LINE}

The BAF beam transport line will consists of a set of magnetic elements (Dipoles, Quadrupoles, Octupoles, and orbit correction Dipoles) to be placed in the BAF tunnel (Fig.1). These elements will transport the slow extracted ion beam[2] from the AGS-Booster and focus it on the BAF target, which will be located $100 \mathrm{~m}$ downstream from the slow-beam extraction point of the AGS Booster.

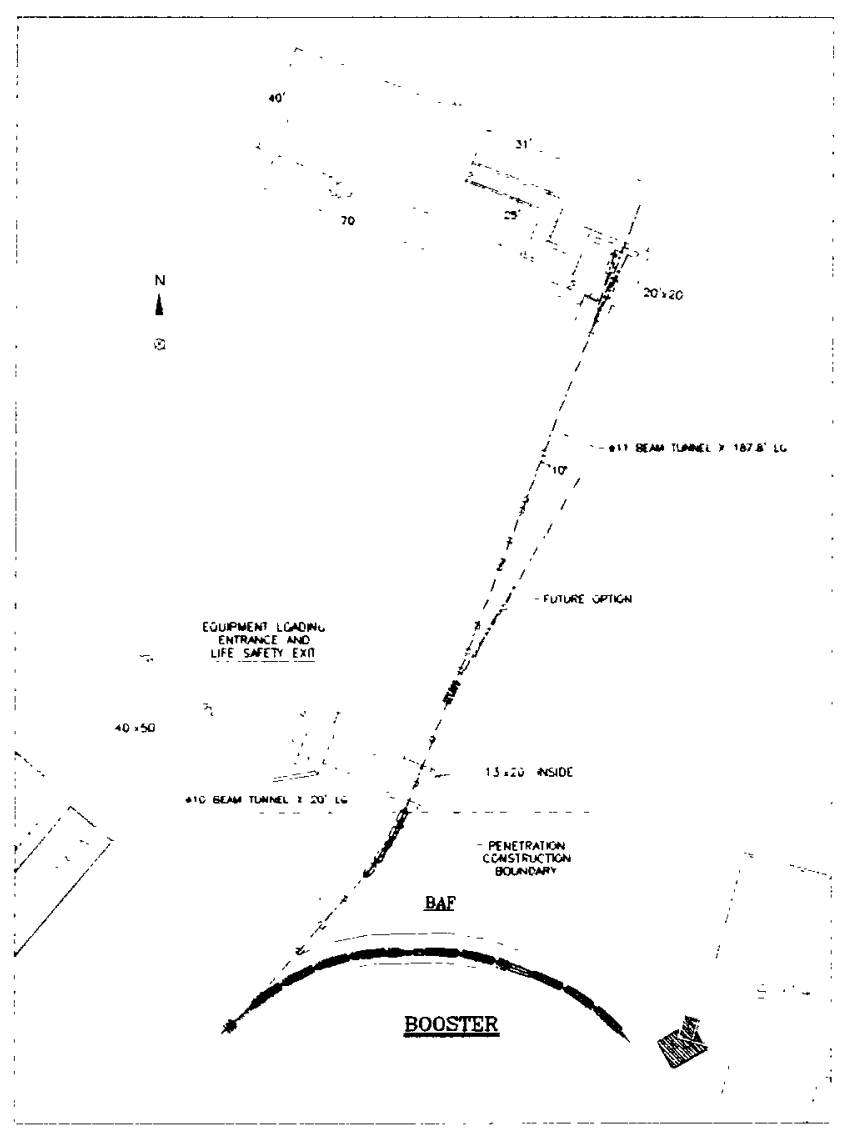

Figure 1: A schematic diagram of the BAF facility with a section of the AGS Booster shown at the bottom if the figure.

The following magnetic elements are used in the beam transport optics of the BAF transport line in the sequence they appear in the line. 
A septum magnet, three quadrupoles, two dipoles $\left(5^{\circ}\right.$ bend each), a quadrupole, two dipoles $\left(5^{\circ}\right.$ bend each), five quadrupoles, an octupole two quadrupoles, an octupole and two quadrupoles. In total there will be five dipoles thirteen quadrupoles and two octupoles. All quads are $40 \mathrm{~cm}$ long and have an aperture of $10 \mathrm{~cm}$ except the last quadrupole which is $50 \mathrm{~cm}$ long and has an aperture of $15 \mathrm{~cm}$. Each of the octupoles is $50 \mathrm{~cm}$ long with an aperture of $10 \mathrm{~cm}$. The septum dipole is $2.3 \mathrm{~m}$ long with a gap of $2.1 \mathrm{~cm}$. Each of the $5^{\circ}$ dipoles is 1.25 $\mathrm{m}$ long with a gap of $8.3 \mathrm{~cm}$.

\section{THE BEAM OPTICS OF (BAF)}

The beam optics of the BAF transport line has been designed to satisfy the following constraints:

a) Transport ion beams with maximum rigidity ${ }^{2}$ $B \rho=13 \mathrm{Tm}$.

b) Transport ion beams with maximum emittances of $\varepsilon_{\mathrm{x}}=3 \pi(\mathrm{mm})(\mathrm{mrad})$ and $\varepsilon_{\mathrm{y}}=15 \pi(\mathrm{mm})(\mathrm{mrad})$ in the horizontal and vertical plane respectively ${ }^{3}$.

c) Generate variable beam sizes at the target, with low and upper limits of $2 \mathrm{~cm}$ to $20 \mathrm{~cm}$ in diameter. These beam spot sizes will include $95 \%$ of the beam.

d) The beam parameters at two locations along the beam line will satisfy certain conditions[1]. These conditions will allow the insertion of octupole magnetic elements which, when turned on, will provide beam spots with rectangular cross section at the target. The beam will be well confined and uniformly distributed over these rectangular areas.

The beam parameters at the beginning of the BAF transport line (see TABLE I) were calculated by modeling the Booster synchrotron[2] using the MAD[3] computer code. In this model the slow extracted beam process was simulated by introducing sextupoles in the Booster lattice, which generated a third order resonance in the beam.

\section{Table I: Beam Parameters at Entrance of BAF}

\begin{tabular}{|l|l|l|l|l|}
\hline & $\beta[\mathrm{m}]$ & $\alpha$ & $\eta[\mathrm{m}]$ & $\eta^{\prime}$ \\
\hline $\operatorname{Hor}(\mathrm{x})$ & 10.0 & 1.87 & 30.0 & -3.4 \\
\hline $\operatorname{Ver}(\mathrm{y})$ & 4.4 & -0.67 & 0.0 & 0.0 \\
\hline
\end{tabular}

The transport system of the BAF transport line, which satisfies the conditions (a) to (d) above, was designed by using the computer codes MAD[3] or TRANSPORT[4].

\footnotetext{
${ }^{2}$ A stripping foil located at the entrance of the BAF line will reduce the rigidity of many charged ions.

${ }^{3}$ The low value of the horizontal emittance is a result of the slow beam extraction process and includes the increase of the emittance when the beam goes through a stripping foil located at the beginning of the BAF transport line, in front of the septum magnet.
}

A particular horizontal/vertical beam envelope along the BAF transport line is shown in Fig. 2. This beam envelope contains $95 \%$ of the beam intensity, corresponds to the emittances and beam parameters shown in Table I and a beam momentum spread $\delta p / p$ $= \pm 0.05 \%$. The beam envelope shown in Fig. 2 corresponds to the lowest momentum that can be extracted from the Booster and thus represents the maximum beam envelope that the BAF line will transport.

The beam spot size on the target can be varied, within the low and upper limits mentioned above, by tuning the strength of the last two quadrupoles of the BAF line.

\section{THIRD ORDER OPTICS}

The first order optics of the BAF transport line, discussed in the previous section, has been designed to allow the placement of two octupoles $\mathrm{O} 1$ and $\mathrm{O} 2$ (see Fig. 2.) at specified locations along the transport line. The beam parameters, at these locations, as calculated from the first order beam optics must satisfy certain conditions[1] for the octupoles to create the desired rectangular and uniform beam spot on the target.

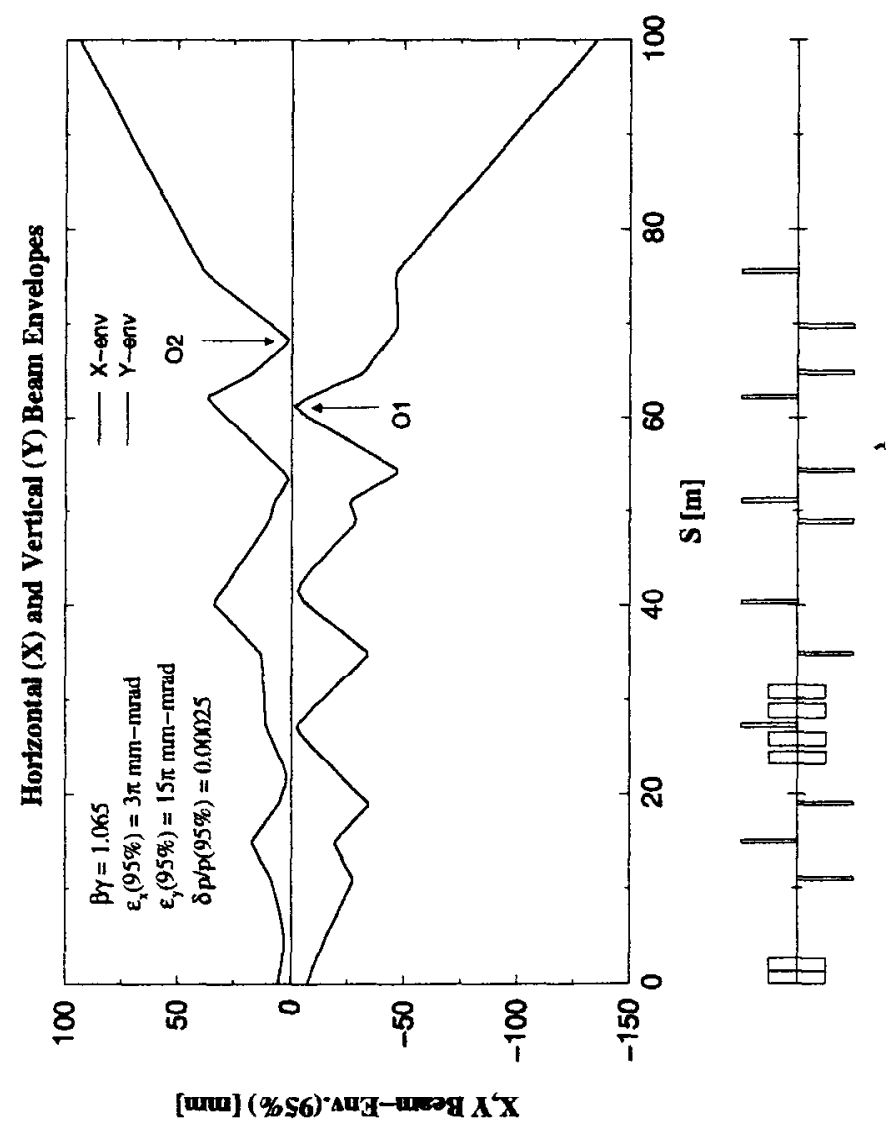

Figure 2: Horizontal and vertical half beam envelopes as calculated using first order beam optics. The beam envelopes contains $95 \%$ of the beam 


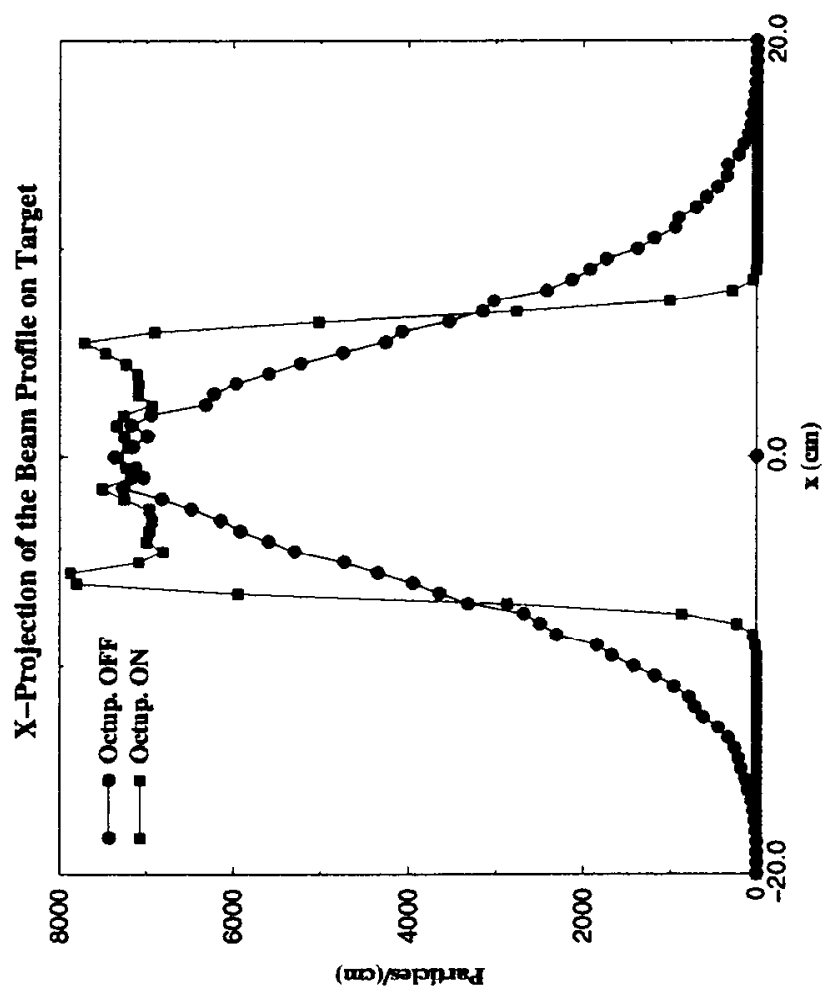

Figure 3a: Horizontal projection of the beam profile on target with the octupole "off" and "on".

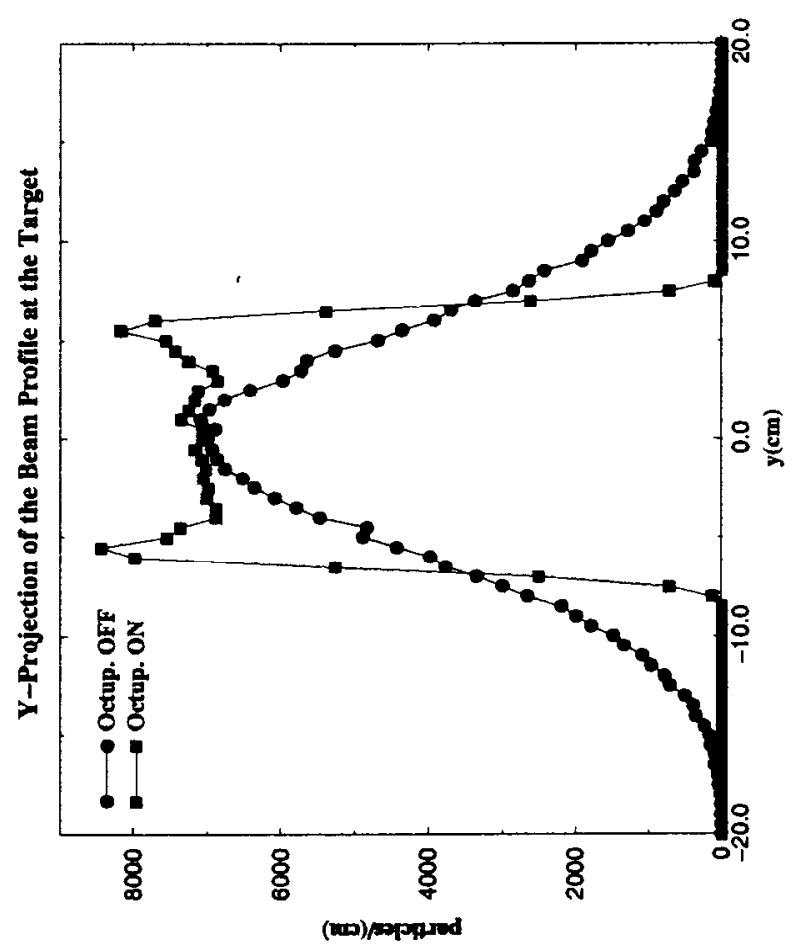

Figure 3b: Vertical projection of the beam profile on target with the octupole "off" and "on".
The third order calculations[1] are required to include the effect of the octupoles on the beam and therefore predict the beam size and its uniformity at the target. For a particular strength of each of 'the octupoles and the last two quadrupoles, a rectangular beam profile is generated at the target. Within this rectangle all beam intensity is confined and distributed rather uniformly over the target. By varying the strength of the above four elements, any rectangular beam spot within the dimensions of $5 \mathrm{~cm}<x<20 \mathrm{~cm}$ and $5 \mathrm{~cm}<y<20 \mathrm{~cm}$ can be generated.

A projection of the horizontal and vertical beam profiles at the target location is shown in Fig. 3a and 3b respectively. Each figure shows the beam profile with the octupoles "off" and the octupoles "on" for comparison purposes.

\section{REMARKS}

A new beam optics for the BAF transport line is being investigated[5] to replace the one presented above. This new beam optics will utilize fewer quadrupoles and dipoles with larger apertures but will retain all the beam requirements discussed earlier.

In the slow extraction process, the beam momentum may vary by $1 \%$ during the cycle of the Booster main magnet, with the beam of lower momentum to be extracted at the end of the extraction cycle. This variation of the beam momentum during the extraction cycle of the Booster can be the cause of beam-spot movement at the BAF target. This beam-spot movement on target can be eliminated by either ramping down the strength of both, the septum magnet and the $20^{\circ}$ dipole or by making achromatic the section of the beam line starting from the septum and finishing at the exit of the $20^{\circ}$ bend.

\section{REFERANCES}

[1] N. Tsoupas, M. S. Zucker, T. E. Ward, and C. L. Snead " Uniform Particle Beam Distributions Produced by Octupole Focusing" Nuclear Sience and Engineering 126, 71 -79 (1997)

[2] K. Brown et. al. "Design of a Resonant Extraction System for the AGS Booster" These Proceedings.

[3] Hans Grote, and F.Christoph Iselin "The MAD Program" CERN/SL/90-13(AP)

[4] D.C. Carey, K.L. Brown, and F. Rothacker "Third-Order Transport a Computer Program for Designing Charged Particle Beam Transport System" SLAC-R-95-462

[5] P. Pile, BNL Private communication 